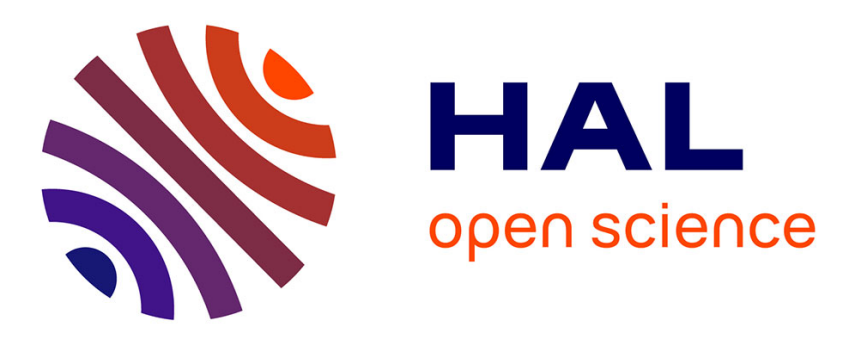

\title{
Mechanistic Learning for Combinatorial Strategies With Immuno-oncology Drugs: Can Model-Informed Designs Help Investigators?
}

Joseph Ciccolini, Dominique Barbolosi, Nicolas André, Fabrice Barlesi, Sébastien Benzekry

\section{To cite this version:}

Joseph Ciccolini, Dominique Barbolosi, Nicolas André, Fabrice Barlesi, Sébastien Benzekry. Mechanistic Learning for Combinatorial Strategies With Immuno-oncology Drugs: Can Model-Informed Designs Help Investigators?. JCO precision oncology, 2020, 108 (4), pp.486-491. 10.1200/PO.19.00381 . hal-03147084

\author{
HAL Id: hal-03147084 \\ https://hal.inria.fr/hal-03147084
}

Submitted on 19 Feb 2021

HAL is a multi-disciplinary open access archive for the deposit and dissemination of scientific research documents, whether they are published or not. The documents may come from teaching and research institutions in France or abroad, or from public or private research centers.
L'archive ouverte pluridisciplinaire HAL, est destinée au dépôt et à la diffusion de documents scientifiques de niveau recherche, publiés ou non, émanant des établissements d'enseignement et de recherche français ou étrangers, des laboratoires publics ou privés. 


\title{
Mechanistic Learning for Combinatorial Strategies With Immuno-oncology Drugs: Can Model-Informed Designs Help Investigators?
}

\author{
Joseph Ciccolini, PharmD, $\mathrm{PhD}^{1}$; Dominique Barbolosi, $\mathrm{PhD}^{1}$; Nicolas André, MD, $\mathrm{PhD}^{1,2}$; Fabrice Barlesi, MD, $\mathrm{PhD}^{3}$; and
} Sébastien Benzekry, $\mathrm{PhD}^{4}$

The past couple of years have seen an unprecedented number of failures of clinical trials investigating combinatorial strategies with immuno-oncology drugs (IODs). Beyond the highly publicized crashes of the MYSTIC study $^{1}$ (anti-PD-L1 plus anti-CTLA4 in non-small-cell lung cancer [NSCLC]) or the ECHO-301 trial ${ }^{2}$ (anti-PD-1 and anti-IDO in melanoma), many other attempts to combine immunotherapy with radiation therapy (RT), ${ }^{3}$ chemotherapy, ${ }^{4}$ metronomic chemotherapy, ${ }^{5}$ or antiangiogenics ${ }^{6}$ have similarly led to disappointing results. Because successful immunotherapy is restricted today to a limited number of patients in a limited number of cancers (melanoma, lung cancer, head and neck cancer, and kidney cancer, with 5-year survival rates $<40 \%$ ), developing appropriate strategies to stretch efficacy remains critical (eg, by turning "cold" tumors [noninflammatory with lack of infiltrating T cells] into "hot" ones [infiltrated by $T$ cells]). Consequently, combining IODs with other treatments likely to harness tumor immunity is appealing. Several studies successfully associated conventional treatments with IODs in comparative phase III trials. ${ }^{7,8}$ However, these trials should not hide the high attrition rate of too many other studies. One of the common characteristics of the trials is the lack of computational pharmacology support, plus the lack of prior knowledge regarding the pharmacokinetics (PK)/ pharmacodynamics (PD) relationships of the combined treatments. In this respect, it is not a surprise that so many studies fail today. Actually, the real surprise comes from the fact that, sometimes, some combinatorial clinical trials manage to be successful.

Author affiliations and support information (if applicable) appear at the end of this article.

Accepted on March 20,2020 and published at ascopubs.org/journal/ po on May 8, 2020: Dol https://doi.org/10. 1200/PO.19.00381
To improve the design of such combinatorial trials beyond trial-and-error methods, we propose an innovative strategy termed mechanistic learning (Fig 1). We define it as the combination of mechanistic modeling-simulation of the kinetics of pathophysiologic processes-and statistical (machine) learning. Using data generated from previous clinical trials and preclinical experiments, it consists in building computational models able to simulate and predict the toxicity and efficacy outcomes of candidate regimens. The optimal scheduling is then selected for clinical trial testing. Importantly, the trial data are then recursively injected in the mechanistic learning process, allowing to further refine predictions. This learning loop allows one to understand which of the biologic premises of the model should be refined or rejected and helps in the next iteration. In analogy to numerical optimization algorithms used in scientific computing (eg, gradient descent), the next trial is thus guided by a rational step, instead of brute-force exploration of alternatives.

\section{THE CURSE OF DIMENSIONALITY: TOO MANY COMBINATIONS TO BE TESTED?}

Combinatorial studies may lead to suboptimal results because of the limited number of arms they explore with respect to the near-infinite number of possible combinations. Limited options when performing combinatorial trials are therefore a major caveat, possibly explaining many failures, or at least limiting the conclusions regarding the real intrinsic potential of a given combination. For instance, in the CheckMate032 study, the anti-PD-1 nivolumab and the antiCTLA4 ipilimumab were administered to patients with NSCLC using only 2 dosing modes (ie, 1 and $3 \mathrm{mg} / \mathrm{kg}$ and vice versa). These doses were chosen because they were already combined in a previous phase I study. ${ }^{9}$ In dose-finding trials, up to 5 dose levels ranging from 0.1 to $20 \mathrm{mg} / \mathrm{kg}$ have been tested for nivolumab and ipilimumab used as single agents without reaching dose-limiting toxicities. ${ }^{10,11}$ Consequently, at least 25 different combinations in dosing could have been explored in CheckMate-032, not to mention the countless variations in sequencing and scheduling. However, without modeling support, only 2 combinatorial arms were tested, thus limiting the chances to find the optimal dose combination. In the subsequent CheckMate-143 study, the same strategy failed to improve survival in patients with glioblastoma. ${ }^{12}$ Another example of a poorly designed study is the MODUL umbrella trial, which tested a triple combination of fluoropyrimidines, the antiangiogenic bevacizumab, and the anti-PD-L1 atezolizumab in patients with metastatic colorectal cancer (mCRC). In theory, a minimum of 12 different sequences should have been explored, not to mention testing different dosing. 


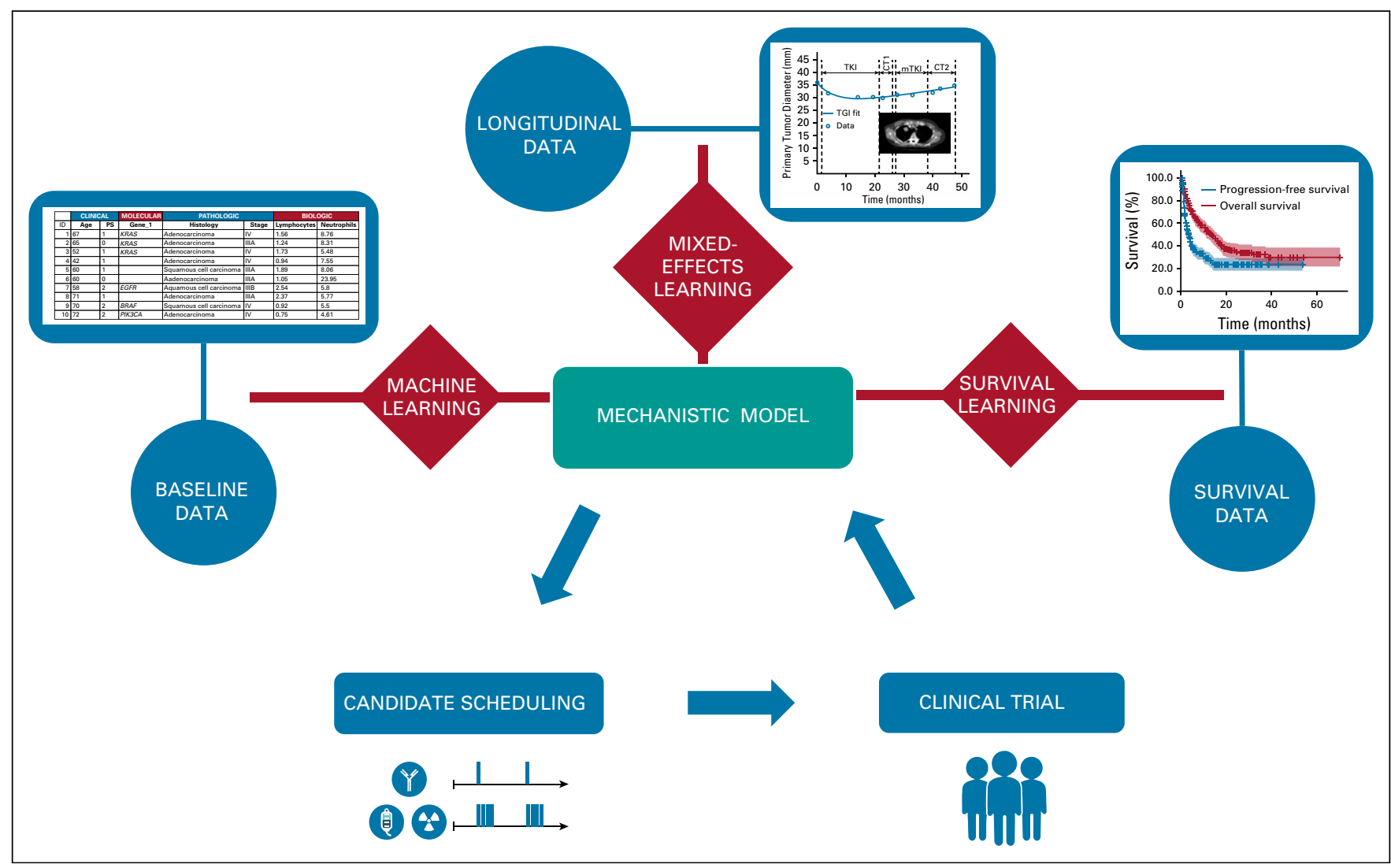

FIG 1. Mechanistic learning. Mechanistic models are mathematical constructs able to simulate pathophysiologic processes. To do so, they depend on mathematical parameters that need to be calibrated from data. Data available in clinical settings are typically of 3 types. Baseline data can be composed of demographic, clinical, pathologic (eg, histologic type), molecular (eg, genetic mutations), or biologic (eg, blood counts) variables. Such covariates can be used as inputs in machine learning algorithms to predict individual values of the parameters. Longitudinal data can include quantities such as tumor size measurements, pharmacokinetics, immune monitoring, seric biomarkers, or circulating DNA. Mixed-effects statistical learning is well adapted to integrate these. Survival data (eg, progression-free or overall survival) can also be modeled with a mechanistic basis (instead of biologically agnostic survival analysis based on, eg, Cox regression), using adapted, survival learning statistical methods. In turn, this allows simulating and optimizing safety and efficacy profiles of candidate therapeutic regimens for combinatorial trials. CT1, first chemotherapy; CT2, second chemotherapy; mTKI, maintenance tyrosine kinase inhibitor; PS, performance status; TGI, tumor growth inhibition; TKI, tyrosine kinase inhibitor.

Surprisingly, in this study, all drugs were administered concomitantly at fixed dosing. This combination failed to exhibit significant efficacy in terms of progression-free survival, and thus, the conclusion was that adding atezolizumab to standard of care for maintenance in mCRC showed no benefit. ${ }^{13}$ Actually, this conclusion may sound peremptory because it is not possible to know whether or not different dosing or scheduling with exactly the same drugs would have performed better. For instance, the combination of the CDK4/6 inhibitor abemaciclib and anti-PD-L1 showed that only slight changes in sequencing (ie, 7-day shift) led to striking differences in antitumor efficacy in mouse models. ${ }^{14}$ Similar critical impact of timing has been demonstrated when combining anti-PD-1 and anti-OX-40 ${ }^{15}$ or when combining RT and IODs. ${ }^{16}$ Sequences could have a major, yet largely underestimated influence on efficacy and should therefore be carefully defined when setting up combinatorial clinical trials with IODs, especially because the immunomodulating properties of cytotoxics are both drug and dose dependent. ${ }^{17}$

\section{ARE PK/PD RELATIONSHIPS REALLY FLAT WITH IMMUNE CHECKPOINT INHIBITORS?}

Although the tumor-mediated drug disposition phenomenon can blur the picture when trying to understand the relationships between drug levels, clearance values, and tumor shrinkage with IODs, exposure-effect relationships have already been evidenced with the anti-CTLA4 ipilimumab, anti-PD-1 nivolumab, and anti-PD-L1 avelumab, to name a few. ${ }^{18-20}$ In this respect, the fact that almost all exploratory trials testing combinatorial strategies lack pharmacokinetics support to evaluate the variability in exposure levels among patients is another major weakness. ${ }^{21,22}$ Before concluding that a combination is ineffective, checking that exposure level with IODs was enough to ensure a correct target engagement could get rid 
of possible confounding factors. Importantly, several flaws can be found even in single-agent studies, blurring the picture when trying next to decipher PK/PD relationships with IODs. For instance, in a pivotal phase I trial of nivolumab, ${ }^{23}$ the impact of dosing (ie, $0.1-10 \mathrm{mg} / \mathrm{kg}$ ) on PD-1 receptor occupancy was investigated. Similar target engagement (ie, 64\%-70\%) was achieved regardless of the dosing, thus prompting several observers to conclude that PK/PD relationships were flat with IODs and, therefore, that interpatient variability was not an issue. However, in this seminal study, PD-1 inhibition was measured only on circulating $T$ lymphocytes extracted from peripheral blood and not on infiltrated T lymphocytes in the tumor microenvironment. Importantly, the pharmacokinetics of most therapeutic monoclonal antibodies is characterized by large interindividual variability and reduced ability to diffuse out of the vascular space and reach solid tumors. ${ }^{24}$ Therefore, to what extent differences in nivolumab dosing could affect or not target engagement at the tumor levels, and not only in circulating T cells, remains to be fully investigated. The once alleged flatness of PK/PD relationships with IODs should therefore not be considered as granted, as suggested by recent pilot studies showing that efficacy in patients with NSCLC is correlated with trough levels of nivolumab and that those are highly variable among patients. ${ }^{20}$

\section{PHARMACOMETRICS IN IMMUNO-ONCOLOGY TO DEFINE DOSING REGIMEN}

Shifting from speculative to model-informed designs is an appealing strategy to reduce the attrition rates during clinical development phases in oncology, where drug candidates still exhibit a $>90 \%$ risk of failure when entering in phase I. ${ }^{25}$ PK/PD modeling is regularly presented as a possible means to improve development of new entities, especially when determining first-in-human doses and recommended phase II doses. ${ }^{26}$ With pembrolizumab, the initial approved dosing was based on such analysis that determined the sufficient dose to ensure meaningful target engagement, ${ }^{27}$ using a quantitative systems pharmacology approach. ${ }^{28}$ Another recent example is the label change for nivolumab from $3 \mathrm{mg} / \mathrm{kg}$ every 2 weeks to $480 \mathrm{mg}$ every 4 weeks, which was also based on a study conducted in silico. ${ }^{29}$

\section{BEYOND PK/PD MODELING: MECHANISTIC LEARNING FOR THE DESIGN OF COMBINATORY CLINICAL TRIALS}

Thus, there is a need for semi-mechanistic models that are able to use the growing amount of quantitative information coming from clinical studies, such as longitudinal data (eg, tumor size, immune biomarkers, or circulating tumor DNA), to create useful simulations of therapeutic regimens (Fig 1 ). Of note, such mechanistic models have started to show their utility in the design of clinical trials, although this has been limited to settings other than immunotherapy thus far. ${ }^{30}$ For instance, the MODEL1 trial (ClinicalTrials.gov identifier: NCT02392845) of a combinatorial regimen in breast cancer and the MetroVino trial (ClinicalTrials.gov identifier: NCT02555007) in lung cancer were both clinical studies entirely driven by mathematical models that identified in silico a complex regimen to be tested at bedside. ${ }^{31-33}$ In the MODEL1 study, neutrophil count monitoring allowed the investigators to perform patient-centered dose individualization so as to limit the incidence of bone marrow toxicity. ${ }^{31,32}$ Such model-informed design could be transposed to preserve immune cells when cytotoxics are to be further associated with IODs.

Other investigators have transposed biologic principles of cancer eco-evolutionary dynamics into mathematical models to understand and predict the onset of resistance to anti-EGFR therapies in lung cancer. ${ }^{34}$ Using models of polyclonal tumor growth, an alternative regimen consisting of a combination of pulse dosing and daily low-dose erlotinib was suggested. ${ }^{35}$ The strategy was further tested in a clinical trial but did not outperform the standard scheme. ${ }^{36} \mathrm{~A}$ likely explanation given by the authors was that peak concentrations did not reach high enough levels in patients in comparison with the in vivo studies. However, interpatient PK variability was not modeled using, for example, mixed-effects statistical techniques. ${ }^{37}$ Combining advanced mathematical modeling with such methods would probably be beneficial. Critically, it would also be highly informative to analyze the data generated using the initial model to close the mechanistic learning loop.

Using similar principles for tumor heterogeneity, others have proposed the concept of adaptive therapy, suggesting individuals be treated only upon disease progression. ${ }^{38}$ This was further successfully translated at bedside in patients with metastatic castration-resistant prostate cancer. ${ }^{39}$ Such a strategy could help to personalize combinations of IODs and aromatase inhibitors in the neoadjuvant treatment of breast cancer. ${ }^{40}$

Despite their scarcity, these studies highlight how application of mathematical modeling in clinical trials in oncology is now feasible and can rationalize study design. These innovative methods are yet to be extended to IOD combinations. Modeling immunotherapy has recently been applied to gain insights on optimal modes for combinations with RT. ${ }^{41-43}$ For instance, Kosinsky et $\mathrm{al}^{42}$ were able to simulate multiple sequences of anti-PD-1 treatment in combination with RT and validated their results using preclinical data. They predicted that hyperfractionated RT regimens had worse efficacy in shrinking tumor size than single-dose regimens. Empirical confirmation or invalidation of such predictions in a clinical setting would be crucial to close the mechanistic learning loop. In another study, a simple model calculating an immunologically effective dose (IED) has been proposed for RT fractionation schemes, ${ }^{44}$ thus suggesting that this kind of algorithm could be used as in silico decision-making tools. 
The general modeling methodology that could be undertaken-mechanistic learning-is a combination of mechanistic modeling and statistical learning, either from machine learning, mixed-effects learning, ${ }^{45}$ or survival learning ${ }^{46}$ for integration of the multimodal data arising from clinical trials or routine management (Fig 1). As output, it would provide informative simulations of the effect of various doses and schedules to aid decisions in early clinical studies. These would consist of predicted probabilities of graded toxicities, tumor growth kinetics in response to treatment, and survival outcomes. Of note, there is a major trend by health regulatory agencies such as the US Food and Drug Administration to call for incorporating extensive modeling and simulation into clinical research. ${ }^{47}$ In this respect, and regarding the current challenges with IODs, ${ }^{48}$ we believe that

\section{AFFILIATIONS}

${ }^{1}$ SMARTc Unit, Centre de Recherche en Cancérologie de Marseille Inserm U1068, Aix Marseille Université, Marseille, France

${ }^{2}$ Pediatric Hematology and Oncology Department, Hôpital Pour Enfant de La Timone, Assistance Publique-Hôpitaux de Marseille, Marseille,

France

${ }^{3}$ Gustave Roussy Cancer Campus, Villejuif, France

${ }^{4}$ MONC Team, INRIA Bordeaux Sud-Ouest and Institut de

Mathématiques de Bordeaux, CNRS UMR5251, Talence, France

\section{CORRESPONDING AUTHOR}

Sebastien Benzekry, PhD, INRIA, 200 Avenue de la Vieille Tour Talence, Nouvelle Aquitaine 33405, France; Twitter: @SBenzekry; e-mail: sebastien.benzekry@inria.fr.

\section{AUTHOR CONTRIBUTIONS}

Conception and design: Joseph Ciccolini, Dominique Barbolosi, Nicolas André, Sébastien Benzekry

Financial support: Fabrice Barlesi

Collection and assembly of data: Joseph Ciccolini, Dominique Barbolosi, Nicolas André, Sébastien Benzekry

Data analysis and interpretation: All authors

Manuscript writing: All authors

Final approval of manuscript: All authors

Accountable for all aspects of the work: All authors

\section{AUTHORS' DISCLOSURES OF POTENTIAL CONFLICTS OF INTEREST}

The following represents disclosure information provided by authors of this manuscript. All relationships are considered compensated unless otherwise noted. Relationships are self-held unless noted. I = Immediate Family Member, Inst = My Institution. Relationships may not relate to the subject matter of this manuscript. For more information about ASCO's mechanistic learning could be a valuable tool for decision making when setting up combinatorial strategies.

\section{CONCLUSION}

After a first phase of enthusiasm, success stories with immunotherapy seem to have reached a glass ceiling because many studies now fail to further stretch either response rates or survival. ${ }^{49}$ Consequently, combining immune checkpoint inhibitors with other treatments likely to boost tumor immunity is a rising strategy in clinical oncology. However, current designs are suboptimal with respect to the complexity of finding the right dosing, scheduling, and sequencing of immune checkpoint inhibitors. We advocate that mechanistic learning could reduce the attrition rate of combinatorial studies through innovative model-informed designs.

conflict of interest policy, please refer to www.asco.org/rwc or ascopubs. org/po/author-center.

Open Payments is a public database containing information reported by companies about payments made to US-licensed physicians (Open Payments).

\section{Joseph Ciccolini}

Honoraria: Pierre Fabre, Pfizer, Roche, Novartis

Research Funding: Roche, Merck Serono

Travel, Accommodations, Expenses: AstraZeneca

Nicolas André

Research Funding: Bristol-Myers Squibb (Inst)

Travel, Accommodations, Expenses: Bristol-Myers Squibb

\section{Fabrice Barlesi}

Honoraria: Genentech, Pfizer, Pierre Fabre, AstraZeneca, Bristol-Myers Squibb, Boehringer Ingelheim, Eli Lilly, Novartis, Merck Serono, MSD Oncology, Takeda, Bayer

Consulting or Advisory Role: Genentech, Pfizer, Novartis, Pierre Fabre, Bristol-Myers Squibb, AstraZeneca/Medlmmune, Boehringer Ingelheim, Eli Lilly, Merck Serono, MSD Oncology, Takeda, Bayer

Research Funding: Genentech (Inst), AstraZeneca/Medlmmune (Inst), Bristol-Myers Squibb (Inst), Pierre Fabre (Inst), AbbVie (Inst), Amgen (Inst), Bayer (Inst), Boehringer Ingelheim (Inst), Eisai (Inst), Eli Lilly (Inst), Ipsen (Inst), Innate Pharma (Inst), Novartis (Inst), Merck Serono (Inst), MSD Oncology (Inst), Pfizer (Inst), Sanofi-Aventis (Inst), Takeda (Inst)

Travel, Accommodations, Expenses: Genentech, Bristol-Myers Squibb, AstraZeneca/MedImmune, MSD Oncology

No other potential conflicts of interest were reported.

\section{ACKNOWLEDGMENT}

We thank Elena Ivanchenko for graphic assistance. Supported by the "Big Bang" Association. In memory of Marie-Christine Masini.

\section{REFERENCES}

1. Rizvi NA, Chul Cho B, Reinmuth N, et al: Durvalumab with or without tremelimumab vs platinum-based chemotherapy as first-line treatment for metastatic nonsmall cell lung cancer: MYSTIC. Ann Oncol 29:10, 2018 (suppl 8; abstr LBA6)

2. Muller AJ, Manfredi MG, Zakharia Y, et al: Inhibiting IDO pathways to treat cancer: Lessons from the ECHO-301 trial and beyond. Semin Immunopathol 41:41-48, 2019

3. McBride SM, Sherman EJ, Tsai CJ, et al: A phase II randomized trial of nivolumab with stereotactic body radiotherapy (SBRT) versus nivolumab alone in metastatic (M1) head and neck squamous cell carcinoma. (HNSCC). J Clin Oncol 36, 2018 (suppl 15; abstr 6009) https://doi.org/10.1200/JCO.2018.36.15_ suppl.6009 
4. Rothschild S, Zippelius A, Savic S, et al: SAKK 16/14: Anti-PD-L1 antibody durvalumab (MEDI4736) in addition to neoadjuvant chemotherapy in patients with stage IIIA(N2) non-small cell lung cancer (NSCLC)—A multicenter single-arm phase II trial. J Clin Oncol 36, 2018 (suppl 15; abstr TPS8584) https://doi.org/10. 1200/JC0.2018.36.15_suppl.TPS8584

5. Toulmonde M, Penel N, Adam J, et al: Use of PD-1 targeting, macrophage infiltration, and IDO pathway activation in sarcomas: A phase 2 clinical trial. JAMA Oncol 4:93-97, 2018

6. Gao J, Karam JA, Tannir NM, et al: A pilot randomized study evaluating nivolumab (nivo) or nivo + bevacizumab (bev) or nivo + ipilimumab (ipi) in patients with metastatic renal cell carcinoma (MRCC) eligible for cytoreductive nephrectomy (CN), metastasectomy (MS) or post-treatment biopsy (Bx). J Clin Oncol 36, 2018 (suppl 15; abstr 4520) https://doi.org/10.1200/JC0.2018.36.15_suppl.4520

7. Socinski MA, Jotte RM, Cappuzzo F, et al: Atezolizumab for first-line treatment of metastatic nonsquamous NSCLC. N Engl J Med 378:2288-2301, 2018

8. Motzer RJ, Penkov K, Haanen J, et al: Avelumab plus axitinib versus sunitinib for advanced renal-cell carcinoma. N Engl J Med 380:1103-1115, 2019

9. Hellmann MD, Rizvi NA, Goldman JW, et al: Nivolumab plus ipilimumab as first-line treatment for advanced non-small-cell lung cancer (CheckMate 012): Results of an open-label, phase 1, multicohort study. Lancet Oncol 18:31-41, 2017

10. Ribas A, Camacho LH, Lopez-Berestein G, et al: Antitumor activity in melanoma and anti-self responses in a phase I trial with the anti-cytotoxic T lymphocyteassociated antigen 4 monoclonal antibody CP-675,206. J Clin Oncol 23:8968-8977, 2005

11. Weber JS, O'Day S, Urba W, et al: Phase I/II study of ipilimumab for patients with metastatic melanoma. J Clin Oncol 26:5950-5956, 2008

12. Filley AC, Henriquez M, Dey M: Recurrent glioma clinical trial, CheckMate-143: The game is not over yet. Oncotarget 8:91779-91794, 2017

13. Grothey A, Tabernero J, Arnold D, et al: Fluoropyrimidine and bevacizumab plus or minus atezolizumab as first-line treatment for BRAF wild type metastatic colorectal cancer: Findings from the MODUL trial of biomarker-driven maintenance. ESMO 2018 Congress, Munich, Germany, October 19-23, 2018 (abstr LBA19)

14. Schaer DA, Beckmann RP, Dempsey JA, et al: The CDK4/6 inhibitor abemaciclib induces a T cell inflamed tumor microenvironment and enhances the efficacy of PD-L1 checkpoint blockade. Cell Rep 22:2978-2994, 2018

15. Messenheimer DJ, Jensen SM, Afentoulis ME, et al: Timing of PD-1 blockade is critical to effective combination immunotherapy with anti-OX40. Clin Cancer Res 23:6165-6177, 2017

16. Young KH, Baird JR, Savage T, et al: Optimizing timing of immunotherapy improves control of tumors by hypofractionated radiation therapy. PLoS One 11:e0157164, 2016

17. Chen G, Emens LA: Chemoimmunotherapy: Reengineering tumor immunity. Cancer Immunol Immunother 62:203-216, 2013

18. Feng Y, Roy A, Masson E, et al: Exposure-response relationships of the efficacy and safety of ipilimumab in patients with advanced melanoma. Clin Cancer Res 19:3977-3986, 2013

19. Heery CR, O'Sullivan-Coyne G, Madan RA, et al: Avelumab for metastatic or locally advanced previously treated solid tumours (JAVELIN Solid Tumor): A phase 1a, multicohort, dose-escalation trial. Lancet Oncol 18:587-598, 2017

20. Basak EA, Koolen SLW, Hurkmans DP, et al: Correlation between nivolumab exposure and treatment outcomes in non-small-cell lung cancer. Eur J Cancer 109:12-20, 2019

21. Hellmann MD, Paz-Ares L, Bernabe Caro R, et al: Nivolumab plus ipilimumab in advanced non-small-cell lung cancer. N Engl J Med 381:2020-2031, 2019

22. Gandhi L, Rodríguez-Abreu D, Gadgeel S, et al: Pembrolizumab plus chemotherapy in metastatic non-small-cell lung cancer. N Engl J Med 378:2078-2092, 2018

23. Topalian SL, Hodi FS, Brahmer JR, et al: Safety, activity, and immune correlates of anti-PD-1 antibody in cancer. N Engl J Med 366:2443-2454, 2012

24. Ryman JT, Meibohm B: Pharmacokinetics of monoclonal antibodies. CPT Pharmacometrics Syst Pharmacol 6:576-588, 2017

25. Beinse G, Tellier V, Charvet V, et al: Prediction of drug approval after phase I clinical trials in oncology: RESOLVED2. JCO Clin Cancer Inform 3:1-10, 2019

26. Garralda E, Dienstmann R, Tabernero J: Pharmacokinetic/pharmacodynamic modeling for drug development in oncology. Am Soc Clin Oncol Educ Book 37:210-215, 2017

27. Chatterjee MS, Elassaiss-Schaap J, Lindauer A, et al: Population pharmacokinetic/pharmacodynamic modeling of tumor size dynamics in pembrolizumabtreated advanced melanoma. CPT Pharmacometrics Syst Pharmacol 6:29-39, 2017

28. Lindauer A, Valiathan CR, Mehta K, et al: Translational pharmacokinetic/pharmacodynamic modeling of tumor growth inhibition supports dose-range selection of the anti-PD-1 antibody pembrolizumab. CPT Pharmacometrics Syst Pharmacol 6:11-20, 2017

29. Long GV, Tykodi SS, Schneider JG, et al: Assessment of nivolumab exposure and clinical safety of $480 \mathrm{mg}$ every 4 weeks flat-dosing schedule in patients with cancer. Ann Oncol 29:2208-2213, 2018

30. Barbolosi D, Ciccolini J, Lacarelle B, et al: Computational oncology: Mathematical modelling of drug regimens for precision medicine. Nat Rev Clin Oncol 13:242-254, 2016

31. Meille C, Barbolosi D, Ciccolini J, et al: Revisiting dosing regimen using pharmacokinetic/pharmacodynamic mathematical modeling: Densification and intensification of combination cancer therapy. Clin Pharmacokinet 55:1015-1025, 2016

32. Hénin E, Meille C, Barbolosi D, et al: Revisiting dosing regimen using PK/PD modeling: The MODEL1 phase I/II trial of docetaxel plus epirubicin in metastatic breast cancer patients. Breast Cancer Res Treat 156:331-341, 2016

33. Barlesi F, Imbs D-C, Tomasini P, et al: Mathematical modeling for phase I cancer trials: A study of metronomic vinorelbine for advanced non-small cell lung cancer (NSCLC) and mesothelioma patients. Oncotarget 8:47161-47166, 2017

34. FooJ, Michor F: Evolution of resistance to targeted anti-cancer therapies during continuous and pulsed administration strategies. PLoS Comput Biol 5:e1000557, 2009

35. Chmielecki J, Foo J, Oxnard GR, et al: Optimization of dosing for EGFR-mutant non-small cell lung cancer with evolutionary cancer modeling. Sci TransI Med 3:90ra59, 2011

36. Yu HA, Sima C, Feldman D, et al: Phase 1 study of twice weekly pulse dose and daily low-dose erlotinib as initial treatment for patients with EGFR-mutant lung cancers. Ann Oncol 28:278-284, 2017

37. Bonate PL: Pharmacokinetic-Pharmacodynamic Modeling and Simulation (ed 2). New York, NY, Springer-Verlag, 2011 https://doi.org/10.1007/978-1-44199485-1

38. Gatenby RA, Silva AS, Gillies RJ, et al: Adaptive therapy. Cancer Res 69:4894-4903, 2009

39. Zhang J, Cunningham JJ, Brown JS, et al: Integrating evolutionary dynamics into treatment of metastatic castrate-resistant prostate cancer. Nat Commun 8:1816, 2017

40. West J, Robertson-Tessi M, Luddy K, et al: The immune checkpoint kick start: Optimization of neoadjuvant combination therapy using game theory. JCO Clin Cancer Inform 3:1-12, 2019 
41. Serre R, Benzekry S, Padovani L, et al: Mathematical modeling of cancer immunotherapy and its synergy with radiotherapy. Cancer Res 76:4931-4940, 2016

42. Kosinsky Y, Dovedi SJ, Peskov K, et al: Radiation and PD-(L)1 treatment combinations: Immune response and dose optimization via a predictive systems model. J Immunother Cancer 6:17, 2018

43. Poleszczuk JT, Luddy KA, Prokopiou S, et al: Abscopal benefits of localized radiotherapy depend on activated T cell trafficking and distribution between metastatic lesions. Cancer Res 76:1009-1018, 2016

44. Serre R, Barlesi F, Muracciole X, et al: Immunologically effective dose: A practical model for immuno-radiotherapy. Oncotarget 9:31812-31819, 2018

45. Claret L, Girard P, Hoff PM, et al: Model-based prediction of phase III overall survival in colorectal cancer on the basis of phase II tumor dynamics. J Clin Oncol 27:4103-4108, 2009

46. Nicolo C, Perier C, Prague M, et al: Machine learning versus mechanistic modeling for prediction of metastatic relapse in breast cancer. JCO Clin Cancer Inform 4:259-274, 2020

47. Goldberg RM, Wei L, Fernandez S: The evolution of clinical trials in oncology: Defining who benefits from new drugs using innovative study designs. Oncologist 22:1015-1019, 2017

48. Coosemans A, Vankerckhoven A, Baert T, et al: Combining conventional therapy with immunotherapy: A risky business? Eur J Cancer 113:41-44, 2019

49. Ledford H: Cancer drug's stumbles prompt calls to rethink how immune therapies are tested. Nature 10.1038/d41586-018-07445-3 